\title{
Réserve nationale stratégique d'urgence du Canada : Une nouvelle stratégie à long terme s'impose
}

\author{
Scott Laing MD, Ellen Westervelt BScS
}

Citation : CMAJ 2020 July 13;192:E810-1. doi: 10.1503/cmaj.200946-f; diffusion hâtive le 25 juin 2020

Voir la version anglaise ici : www.cmaj.ca/lookup/doi/10.1503/cmaj.200946

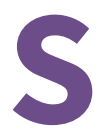

elon de récents rapports publiés dans les médias, des millions de pièces d'équipement de protection individuelle (ÉPI) de la Réserve nationale stratégique d'urgence (RNSU) ont atteint leur date de péremption et se trouvent gaspillées ${ }^{1}$. Les gouvernements et des établissements de santé essaient également de se constituer rapidement de nouveaux stocks pour répondre aux nombreuses pénuries ${ }^{2}$. La gestion des réserves stratégiques a connu des ratés au Canada et ailleurs dans le monde, y compris aux États-Unis, en Australie, particulièrement en ce qui concerne les dates de péremption et le gaspillage financier ${ }^{3,4}$. En nous inspirant de la réponse à la pandémie et des audits des réserves d'urgence d'autres pays, nous proposons ici une nouvelle stratégie à long terme pour gérer la Réserve nationale stratégique d'urgence tout en réduisant le gaspillage et en assurant des approvisionnements adéquats.

La RNSU a été créée en 1952 durant la Guerre froide; elle comportait alors un hôpital mobile et des fournitures dites de "services sociaux » pour pouvoir réagir en cas de catastrophe nucléaire ${ }^{5}$. Après quelques désastres, ici et ailleurs, le RNSU a évolué pour inclure plus généralement de l'équipement médical, des médicaments et des fournitures de services sociaux ${ }^{5}$. C'est après la pandémie de syndrome respiratoire aigu sévère (SRAS) en 2003 que l'Agence de la Santé publique du Canada (ASPC) a été mise sur pied et les actifs de la RNSU sont alors passés de Santé Canada à l'ASPC 5 . La pandémie de SRAS est aussi à l'origine de la Loi sur la gestion des urgences ${ }^{6}$ et du Plan fédéral d'intervention d'urgence ${ }^{7}$, en vertu duquel le gouvernement du Canada doit assurer la coordination des mesures d'urgence «tous risques »,7.

En date du dernier audit public de la RNSU (2010), la valeur estimée de tous ses actifs s'élevait à 300 millions de dollars, ses budgets annuels de fonctionnement à 4 millions de dollars et la location annuelle des entrepôts à 7,7 millions de dollars ${ }^{5}$. Les stocks étaient conservés dans 11 entrepôts au pays et avaient été utilisés à 128 reprises au cours des 25 années précédentes ${ }^{5}$. Le problème de péremption des stocks a été soulevé lors de

\section{POINTS CLÉS}

- La pandémie de maladie à coronavirus 2019 a mis en lumière les problèmes qui affectent la chaîne d'approvisionnement en équipement de protection individuelle au Canada et ailleurs dans le monde.

- Le dernier audit de la Réserve nationale stratégique d'urgence (RNSU) du Canada remonte à 2010; un problème de péremption des fournitures a été noté à l'époque et persiste encore aujourd'hui.

- Les réserves de fournitures d'urgence sont coûteuses à maintenir, et la gestion des fournitures périmées génère un gaspillage substantiel d'argent et de matériel.

- Une meilleure approche pourrait être d'intégrer la RNSU avec l'approvisionnement commercial par le biais d' un « fournisseur principal », ce qui réduirait le gaspillage d'argent et de matériel par la vente de fournitures directement aux établissements de santé; cela assurerait la fraîcheur des réserves, permettrait de maintenir un approvisionnement adéquat de matériel non périmé et fournirait d'importantes données sur l'offre et la demande lors de crises.

l'audit de la RNSU de 2010; on a découvert que certaines fournitures dataient des années $1960^{\circ}$. Les plans d'entretien actuels de la réserve prévoient le déploiement des stocks ou leur élimination, mais de nombreuses fournitures atteignent leur date de péremption avant de pouvoir être utilisées parce qu'elles ne sont déployées qu'après épuisement des stocks antérieurs. Selon les reportages dans les médias ${ }^{8}$, la réserve du Canada contient encore beaucoup de fournitures périmées ${ }^{1}$. Les quantités exactes disponibles sont également inconnues ${ }^{1}$ parce que la RNSU est dépourvue de tout système électronique de gestion des stocks et les données les plus récentes datent de l'audit de $2010^{8}$. Le gouvernement du Canada a du mal actuellement à assurer la coordination rapide de ses approvisionnements en nouvelles fournitures d'ÉPI ${ }^{2}$ en raison des retards de livraison des commandes, 
ce qui avait déjà été mentionné lors de l'audit de $2010^{8}$. Les pénuries d'ÉPI qui en résultent ont retardé l'approvisionnement de certains services de santé, particulièrement dans la communauté, où plusieurs médecins de famille ont eu un accès limité à des fournitures d'ÉPI ${ }^{9}$ essentielles. Ces fournitures peuvent être achetées auprès de sociétés tierces, mais cela devient difficile quand la demande augmente.

Après la pandémie de grippe A ( $\mathrm{H} 1 \mathrm{~N} 1)$ en 2009, une analyse américaine a relevé le problème des chaînes d'approvisionnement en ÉPI ${ }^{10}$. Plusieurs organisations ont substantiellement gonflé leurs commandes pour se constituer des réserves et lorsque les commandes ont été annulées ou servies partiellement, d'autres ont été placées auprès de plusieurs fournisseurs. Quand le gouvernement a essayé d'aider à coordonner une réponse, la situation s'est considérablement envenimée; faute de de système de commande centralisé pour générer des données cohérentes, il était impossible de prédire l'offre et la demande et les fournisseurs privés résistaient à partager leurs données par crainte de la concurrence ${ }^{10}$. Après ces écueils, on a recommandé de faire le suivi de l'utilisation des fournitures d'ÉPI et de centraliser les données relatives aux commandes ${ }^{10}$.

Tout comme le Canada, les États-Unis maintiennent leur réserve nationale stratégique (SNS) pour coordonner les mesures d'urgence ${ }^{3}$. Un coup d'œil à la SNS a aussi révélé des dépenses élevées dues à la péremption des fournitures avant leur utilisation ${ }^{3}$. Pour réduire l'impact de la péremption des stocks, les États-Unis ont choisi de prolonger les dates de péremption au-delà des dates affichées par les fabricants ${ }^{3}$. Même si cette approche peut être efficace à court terme, elle n'a pas résolu le problème des dates de péremption ${ }^{3}$.

Un audit de la réserve médicale nationale australienne (ANMS) a aussi révélé un gaspillage très coûteux ${ }^{4}$. Au cours des 10 années précédant cet audit, les Australiens ont investi 750 millions de dollars dans leur ANMS. Là-dessus, près de 250 millions de dollars en fournitures périmées ont généré des coûts d'élimination de 75 millions de dollars ${ }^{4}$. La stratégie d'entretien de la réserve australienne repose sur une rotation des stocks ${ }^{4}$, le retour des stocks périmés et la prolongation des durées de conservation.

Il est plus que temps que le Canada se dote d'une nouvelle stratégie à long terme à cet égard en utilisant les données sur l'offre et la demande ${ }^{10}$, et qu'il s'assure que les fournitures soient utilisées avant leur péremption ${ }^{3,4,8}$ en suivant un modèle financièrement responsable $e^{3,4,8}$.

Selon une approche, on pourrait demander à tous les fournisseurs de faire rapport des commandes reçues à une agence centrale ${ }^{10}$ qui pourrait coordonner plus efficacement l'utilisation des réserves et envoyer un signal pour un cycle de fabrication anticipé ${ }^{10}$. Selon une autre approche, on pourrait développer davantage de programmes d'élimination ${ }^{8}$ et de prolongation ${ }^{3}$ des durées de conservation ${ }^{4}$. Toutefois, ni l'une ni l'autre de ces options ne résoudrait le problème de péremption des stocks et le gaspillage qui en résulte ${ }^{3,4}$.

Une meilleure approche serait d'intégrer la RNSU avec l'approvisionnement commercial ${ }^{10}$. L'intégration pourrait être réalisée par l'entremise d'un "fournisseur principal », une société de la Couronne ou un organisme à but non lucratif ${ }^{4}$. Ce fournisseur principal gèrerait l'inventaire, l'approvisionnement, les négociations de contrat, les rapports sur les données, la logistique et la distribution ${ }^{4}$. Il pourrait emprunter le modèle des organisations de services partagés (OSP) qui fournissent des approvisionnements par le biais de contrats d'achats groupés pour faire des économies d'échelle et assurer la stabilité et l'équité des prix ${ }^{11}$. Le fournisseur principal maintiendrait les entrepôts pleins et vendrait les fournitures aux hôpitaux, aux cliniques privées, aux établissements de soins de longue durée pour une utilisation de routine. Ainsi, les stocks seraient toujours frais et l'approvisionnement suffisant pour répondre aux situations d'urgence. Le gouvernement du Canada pourrait utiliser les données sur l'offre et la demande provenant des ventes pour mieux coordonner sa réponse aux situations d'urgence et prévoir les pénuries afin de déclencher un cycle de fabrication anticipée ${ }^{10}$. On pourrait vérifier régulièrement les types de fournitures en réserve pour s'assurer de leur pertinence. Et si cette approche est efficace, le fournisseur principal pourrait éventuellement diversifier ses opérations et inclure des produits de soins aigus et des médicaments.

Nous reconnaissons que l'approche proposée, avec fournisseur principal, ne résoudra pas les pénuries actuelles ${ }^{1}$ ni les problèmes d'approvisionnement internationaux ${ }^{2}$. Elle aurait ses propres inconvénients, entre autres, le choix des fabricants, l'optimisation de la taille des réserves, la logistique de gestion de l'inventaire et les obstacles bureaucratiques. Un fournisseur principal pourrait donc commencer à petite échelle et croître avec le temps.

La péremption des stocks est un défi important pour les réserves nationales. Il est donc urgent de trouver une nouvelle solution à long terme pour gérer la chaîne d'approvisionnement en ÉPI. Vendre et renouveler les stocks pour maintenir un stock minimum en cas d'urgence permettrait d'assurer un approvisionnement adéquat et réduirait le gaspillage de ressources. Le Canada doit agir maintenant pour mieux se préparer à la prochaine crise.

\section{Références}

1. Leo G. Health minister reviewing management of Canada's emergency stockpile. CBC News le 15 avril 2020, mis à jour le 16 avril 2020. Accessible ici : www.cbc.ca/ news/canada/saskatchewan/heath-minister-emergency-stockpile-1.5530081 (consulté le 16 avril 2020).

2. Cochrane D, Harris K. Canada building its own PPE network in China. CBC News le 13 avril 2020, mis à jour le 16 avril 2020. Accessible ici : www.cbc.ca/news/ politics/canada-building-own-ppe-supply-chain-in-china-1.5530259 (consulté le 16 avril 2020)

3. The strategic national stockpile: origin, policy foundations, and federal context. In: The Nation's Medical Countermeasure Stockpile: opportunities to improve the efficiency, effectiveness, and sustainability of the CDC Strategic National Stockpile: workshop summary. Washington (DC) : National Academies Press (É.-U.); 2016. Accessible ici : www.ncbi.nlm.nih.gov/books/NBK396378 (consulté le 19 avril 2020).

4. Management of the National Medical Stockpile. Auditor - general report No 53 of 2013-14. Canberra (AU): Department of Health, Australian National Audit Office; 2014.

5. Section 2 : Évaluation du système de la réserve nationale d'urgence (SRNU) Contexte. Ottawa : Agence de la santé publique du Canada; modifié le 28 août 2012. Accessible ici : www.canada.ca/fr/sante-publique/organisation/ mandat/a-propos-agence/bureau-evaluation/rapports-evaluation/evaluation -systeme-reserve-nationale-urgence/contexte.html (consulté le 16 avril 2020). 
6. Loi sur la gestion des urgences (L.C. 2007, ch. 15). Accessible ici : laws-lois.justice .gc.ca/fra/lois/e-4.56/page-1.html (consulté le 17 avril 2020).

7. Plan fédéral d'intervention d'urgence - janvier 2011. Ottawa : Sécurité publique Canada; modifié le 31 janvier 2018. Accessible ici : www.securitepublique. gc.ca/cnt/rsrcs/pblctns/mrgnc-rspns-pln/index-fr.aspx (consulté le 17 avril 2020).

8. Section 3: Évaluation du système de la réserve nationale d'urgence (SRNU) Résultats. Ottawa : Agence de la santé publique du Canada; modifié le 28 août 2012. Accessible ici : www.canada.ca/fr/sante-publique/organisation/ mandat/a-propos-agence/bureau-evaluation/rapports-evaluation/evaluation -systeme-reserve-nationale-urgence/resultats.html (consulté le 16 avril 2020).

9. Kupfer M. Mask shortage threatening family practices, doctors say. CBC News le 31 mars 2020. Accessible ici : www.cbc.ca/news/canada/ottawa/mask-shortage -threatening-family-practices-1.5511360 (consulté le 17 avril 2020).

10. Patel A, D'Alessandro MM, Ireland KJ, et al. Personal protective equipment supply chain: lessons learned from recent public health emergency responses. Health Secur 2017;15:244-52.

11. Le partage de services appliqué à la santé : Analyse prospective. Ottawa : Agence canadienne des médicaments et des technologies de la santé; 2011. www.cadth.ca/media/pdf/Health_SS_es_24_f.pdf (consulté le 17 avril 2020).
Intérêts concurrents : Aucun déclaré.

Cet article a été révisé par des pairs.

Affiliations : Département de médecine familiale (Laing), Université d'Ottawa; Approvisionnement (Westervelt), Hôpital Queensway Carleton, Ottawa, Ont.
Collaborateurs : Scott Laing s'est chargé de l'élaboration, de la recherche de base et de la rédaction de l'article. Ellen Westervelt s'est chargée de passer l'article en revue, d'en formuler une critique constructive et de le réviser. Les deux auteurs en ont approuvé la version définitive pour publication et ont accepté de se rendre responsables de tous les aspects du travail.
Avis : Les opinions exprimées dans ce commentaire n'engagent que les auteurs et ne reflètent pas nécessairement l'opinion de l'Hôpital Queensway Carleton.

Correspondance : Scott Laing, slain027@uottawa.ca 